Originalien

Nervenarzt 2021 · 92:243-251

https://doi.org/10.1007/s00115-020-01056-y

Angenommen: 9. Dezember 2020

Online publiziert: 12. Januar 2021

๑ Der/die Autor(en) 2021

\section{Die globale Ausbreitung und in- dividuelle Bedrohung durch die COVID-19-Pandemie und die zur Be- wältigung dieser Krise getroffenen gesetzlichen Maßnahmen stellen jede*n Einzelne*n vor individuelle psychische Herausforderungen. So- wohl die Angst vor einer Ansteckung mit dem Virus oder der Erkrankung geliebter Menschen als auch die Fol- gen der Hygienemaßnahmen mit Kontaktbeschränkungen bis hin zur Isolation sind sehr belastend und lassen allgemein die Entwick- lung psychischer Erkrankungen befürchten. In diesem Beitrag wer- den die psychischen Konsequenzen für psychisch erkrankte Personen untersucht.}

Psychische Folgen individueller lebensbedrohlicher Situationen sind vorbeschrieben [37, 43]. Ebenso sind psychische Erkrankungen als Folge kollektiver und globaler Bedrohungen bekannt - z. B. bei Naturkatastrophen [33], im Krieg [16] oder auf der Flucht [18]. In dieser aktuellen Pandemie werden weltweit vermehrt psychische Erkrankungen beobachtet. In China wurden bei 12- bis 18-jährigen Jugendlichen hohe Raten an krankheitswertiger ängstlicher (43,7\%) und depressiver $(37,4 \%)$ Symptomatik

Johanna G. Winkler' • Dario Jalilzadeh Masah' - James Kenneth Moran ${ }^{2}$. Joachim Bretz ${ }^{1}$ loannis Tsagkas ${ }^{1}$ - Thomas Goldschmidt ${ }^{1}$ - Meryam SchoulerOcak $^{1}$

' Psychiatrische Institutsambulanz der Charité im St. Hedwig-Krankenhaus, Charité - Universitätsmedizin Berlin, Berlin, Deutschland

${ }^{2}$ Research Group Multisensory Integration der Charité im St. Hedwig-Krankenhaus, Charité Universitätsmedizin Berlin, Berlin, Deutschland

\title{
Psychische Belastung während der COVID-19-Pandemie: Konsequenzen für psychiatrisch Erkrankte und therapeutische Implikationen
}

gemessen, wobei beides negativ assoziiert war mit fachlichem Wissen über das Virus [42]. Auch krankheitswertige Symptome einer posttraumatischen Belastungsstörung (PTSD) wurden beobachtet - in Italien [7] und China [40] bei rund einem Drittel der Allgemeinbevölkerung, in den USA bei 31,8\% [20]. Frauen sind besonders belastet $[7,8]$.

In der COVID-19-Pandemie hat soziale Isolation einen schützenden Effekt bezüglich Ansteckungswahrscheinlichkeit und Mortalität und somit für die physische Gesundheit [26]. In der Allgemeinbevölkerung bewirkt soziale Isolierung im psychischen Bereich eine Zunahme von depressiven, Angst- und Stresssymptomen sowie Ärger und wirkt u. U. stigmatisierend [28].

Für einzelne Personengruppen wurde die psychische Vulnerabilität in dieser Situation bereits gesondert betrachtet. In Kanada wird die besondere klinische Beobachtung schwangerer Frauen angedacht, die aktuell wesentlich mehr mit ängstlich-depressiven, dissoziativen und posttraumatischen Symptomen belastet sind als vor der Pandemie [3]. Weltweit wurde vermehrt psychisch und physisch traumatisierende häusliche Gewalt als unmittelbare Folgen eines Lockdowns beobachtet $[13,22]$; infolgedessen werden bereits spezielle Schutzmaßnahmen für Frauen [27] und Kinder [21] gefordert. Auch Gesundheitsbeschäftigte wurden spezifisch untersucht [25], insbesondere das auf Intensivstationen tätige Personal, das schon in Vor-Corona-Zeiten psychisch sehr stark beansprucht war [14].

Eine weitere, bislang wenig beachtete, für psychische Belastung sehr gefährdete Gruppe der Bevölkerung ist die der psychisch Vorerkrankten. Bei ihnen besteht eine signifikant stärkere und häufigere Entwicklung von u. a. Suizidgedanken, PTSD-Symptomatik und Schlafstörungen als bei der psychisch gesunden Allgemeinbevölkerung [15]. Unter diesen sind die älteren Menschen mit somatischen Komorbiditäten und die sozioökonomisch Gefährdeten besonders betroffen [6]. Eine Zunahme von Alkoholrückfällen und Wahnsymptomatik wurde vermutet $[17,31,35]$.

Die psychiatrische Akutversorgung müsse sich auf die Behandlung schwer psychisch erkrankter SARS-CoV-2-Infizierter vorbereiten [12], als Reaktion auf die täglich wechselnden Hygienemaßnahmen bestünde die Notwendigkeit, "fast täglich die Psychiatrie neu“ zu „erfinden“ [31], ähnlich wie in der Psychiatrischen Universitätsklinik der Charité im St. Hedwig-Krankenhaus umgesetzt (PUK der Charité im SHK; 
[17]). Faktisch bedeutete die COVID-19Pandemie-bedingte Umstrukturierung der psychiatrischen Akutstationen, dass mehr schwer psychisch Erkrankte auf weniger stationäre Behandlungsplätze trafen und daher ambulant mitbehandelt werden mussten. Dies in einer Zeit, in der komplementäre ambulante Betreuungsangebote (z.B. Tageskliniken, Tagesstätten, Werkstätten, Einzelfallhilfe, Soziotherapie, ergotherapeutische Gruppen) zum großen Teil nicht zur Verfügung standen [10].

Wir nahmen an, dass die von uns behandelten psychisch erkrankten Menschen im Rahmen der COVID-19-Pandemie erheblich psychisch belastet sein würden, wie dies andere Studien sowohl für die Allgemeinbevölkerung [7, 20, 40, 42] sowie auch für die psychisch vorerkrankte Bevölkerungsgruppe beschrieben $[6,15]$. Wir erwarteten diagnoseabhängig unterschiedliche Aussagen bezüglich einer Belastung durch Infektionsangst oder die Folgen der Schutzmaßnahmen sowie eine diagnoseabhängige Prävalenz beobachteter klinischer Symptomatik. Dabei erwarteten wir zudem vermehrte Trinkrückfälle bei Alkoholabhängigen und Wahnsymptomatik bei schizophren Erkrankten [35], aber auch wie in anderen Studien vorbeschrieben eine stärkere Zunahme von Angst, Depression, Ärger, Impulsivität, Schlafstörungen, Suizidgedanken [9] und PTSDSymptomatik [7, 15, 20, 40]. Ebenso vermuteten wir eine höhere Belastung bei älteren Behandelten [6] und Frauen [7, 8].

Wirbildeten die Hypothese, dass es sowohl für die klinische Verschlechterung der Symptomatik als auch für die subjektive Wahrnehmung der belastenden Ursache diagnoseabhängige Unterschiede geben wird.

\section{Methodik}

In einer retrospektiven Analyse untersuchten wir die Dokumentationen in Krankenakten aller von uns während der Berliner Lockdown-Maßnahmen im Zeitraum vom 01.04.2020 bis zum 30.04.2020 ambulant Behandelten hinsichtlich Geschlecht und Alter, psychischer Erkrankungen, der klinischen
Veränderung ihrer Krankheitssymptomatik, der subjektiv wahrgenommenen Ursache dieser Veränderung und der von uns getroffenen therapeutischen Maßnahmen. Dabei berücksichtigen wir die von uns in einem Pflegeheim Behandelten nicht, da sehr viele von diesen krankheitsbedingt nicht mehr zur differenzierten verbalen Kommunikation fähig waren.

Bei den Daten der vorliegenden Studie handelt es sich um eine Sekundärdatenanalyse $[2,32,41]$ der vorliegenden Krankenhausdaten der Psychiatrischen Institutsambulanz der Charité im SHK Berlin. Die Krankheitssymptomatik der behandelten Patient ${ }^{\star}$ innen wird nicht nur einmalig, sondern bei jedem ihrer Besuche in der psychiatrischen Institutsambulanz dokumentiert.

Damit liegen nicht alleine Daten während der Berliner Lockdown-Maßnahmen im eigentlichen Beobachtungszeitraum vom 01.04.2020 bis zum 30.04.2020 vor, sondern auch Daten für den Zeitraum vor Beginn der Lockdown-Maßnahmen. Daher kann die Veränderung der Symptomatik erfasst werden, indem die Daten vor den Lockdown-Maßnahmen mit den Daten während der Lockdown-Maßnahmen verglichen werden. Die vorliegenden Daten (vor Lockdown, während Lockdown) wurde von unserer Arbeitsgruppe geratet [4]. Unterschieden wurden drei Kategorien, in denen sich die Intensität der klinischen Verschlechterung bei einem Patienten abbildet. Unterschieden wurde, ob sich "keine", eine „leichte“ bzw. eine „schwere“ Verschlechterung zeigt. Standardisierte Fragebögen wurden nicht verwendet, da sich die dafür erforderlichen detaillierteren Aspekte nicht extrahieren ließen.

Dabei wurden die in der von den Behandelnden in der Krankenakte dokumentierten pathologischen Verhaltensänderungen - wie z.B. starker sozialer Rückzug, Suizidimpulse oder -handlungen, stärkeres Konsumverhalten - als "schwere klinische Verschlechterung" gewertet, während die ausschließlich im pathologischen Befund beschriebenen Verschlechterungen - wie z.B. Zunahme des Grübelns, Auftreten von Suizidgedanken usw. - als "leichte klinische Verschlechterung“ gewertet wur- den. Standardisierte Fragebögen wurden nicht verwendet. Die Äußerungen der Patient*innen bezüglich der subjektiv wahrgenommenen Belastung wurden in den Kategorien „nicht belastet“, „Belastung durch die Angst im Zusammenhang mit SARS-CoV-2 und COVID19 (,durch Angst vor Virus') “, „Belastung durch eine der direkten oder indirekten Konsequenzen der gesetzlich implementierten Hygienemaßnahmen (,durch Maßnahmen')“, „es geht mir besser als sonst (,es geht mir besser')", „Belastung sowohl durch Angst vor Infektion als auch durch Maßnahmen (,durch beides') ", , andere, nicht durch die Pandemie bedingte Belastung (,durch etwas anderes') “ oder "fehlende Angabe“ unterschieden.

Die therapeutischen Maßnahmen wurden von den psychiatrisch erfahrenen Behandelnden, ggf. unter Supervision, entschieden, die Diagnosen nach ICD-10 gestellt. Da sich die Haupt- und Nebendiagnosen systembedingt nicht unterscheiden ließen, berücksichtigten wir pro Patient*in bis zu zwei Diagnosen unterschiedlicher Syndromzugehörigkeit (häufbare Merkmale) in unserer Bewertung, sodass ein depressiver, alkoholabhängiger Patient sowohl im Syndromcluster "Abhängigkeit" als auch im Cluster „depressives Syndrom“ berücksichtigt wurde. Für die so gewonnenen Diagnosegruppen errechneten wir mittels $\chi^{2}$-Test nach Pearson Belastungsart und Belastungsstärke und verglichen diese miteinander.

Die statistischen Analysen der erhobenen Daten wurden mit der Software GNU pspp 0.10.2-g654fff und mit IBM SPSS Statistics 25.0 und R 3.6.3 durchgeführt. Für die Analyse aller nominalen Variablen (wie Belastungsart und -stärke, Diagnose, ergriffene Maßnahme oder Geschlecht) wurden Pearson's $\chi^{2}$-Tests verwendet. Bei kleinen Fallzahlen wurden der exakte $\chi^{2}$-Test als Monte-Carlo-Variante mit einer $99 \%$ igen Signifikanz und ein exakter Test nach Fisher ${ }^{[* \mathrm{~F}]}$ eingesetzt. Kritische Signifikanzwerte wurden nach Benjamini und Hochberg (1995) für multiple Vergleiche über alle Analysen hinweg angepasst ${ }^{\left[{ }^{*} \mathrm{BH}\right]}$. Die Wirkung kardinalskalierter unabhängiger Variablen (wie Alter und Zahl der Kontakte und 
Nervenarzt 2021 · 92:243-251 https://doi.org/10.1007/s00115-020-01056-y

(c) Der/die Autor(en) 2021

\section{J. G. Winkler · D. Jalilzadeh Masah · J. K. Moran · J. Bretz · I. Tsagkas · T. Goldschmidt · M. Schouler-Ocak}

\section{Psychische Belastung während der COVID-19-Pandemie: Konsequenzen für psychiatrisch Erkrankte und therapeutische Implikationen}

\section{Zusammenfassung}

Hintergrund. Der Ausbruch der COVID-19-

Erkrankung und die rasche Ausbreitung des sie verursachenden Coronavirus SARS-CoV-2 bedroht weltweit nicht nur die physische, sondern auch die psychische Gesundheit der Bevölkerung. Seine Auswirkungen auf Neuerkrankungen und die Entwicklung bestehender Erkrankungen im ambulanten psychiatrischen Bereich in Deutschland ist noch nicht untersucht.

Methoden. Die Dokumentation in den Akten von 682 behandelten psychisch erkrankten Personen wurde retrospektiv bezüglich ihrer subjektiv erlebten Belastung durch die Pandemie und der klinischen Relevanz hin untersucht.
Ergebnisse. Bei $60,5 \%(n=378)$ bestand eine psychische Belastung durch diese Pandemie. $14,5 \%(n=99)$ der Betroffenen litten unter Angst vor dem Virus SARS-CoV-2 und einer möglichen Infektion, 25,5\% $(n=174)$ unter den getroffenen Schutzmaßnahmen (Lockdown) und 4,3\% $(n=29)$ unter beidem; hierbei bestanden signifikante diagnoseabhängige Unterschiede. Angsterkrankte waren signifikant stärker belastet und hatten mehr Angst vor der Erkrankung, Psychoseerkrankte waren signifikant weniger belastet. Bei $43,7 \%$ aller dieser Belasteten ( $n=132)$ wurde eine akute therapeutische Intervention erforderlich, 6,0\% $(n=18)$ mussten stationär eingewiesen werden.
Diskussion. Psychisch Vorerkrankte gehören zu den durch die Belastungen der COVID19-Pandemie besonders gefährdeten Bevölkerungsgruppen. Langfristigere Untersuchungen zum Verlauf der psychischen Belastungen unter Pandemiemaßnahmen sowie Studien zur Förderung von Resilienz in dieser Bevölkerungsgruppe und die Implementierung solcher Maßnahmen sind erforderlich.

Schlüsselwörter COVID-19-Pandemie · Psychische Erkrankung · Psychische Belastung · Lockdown . Krisenintervention

\section{Psychological stress during the COVID-19 pandemic: consequences for psychiatric patients and therapeutic implications}

Abstract

Background. The outbreak of the COVID-

19 disease and the rapid spread of the inducing coronavirus SARS-CoV-2 threatens not only people's physical health but also their mental health. Its influence on incidence and course of existing illnesses in the psychiatric outpatient sector in Germany is still unknown. Methods. The medical reports of 682 persons in psychiatric treatment were retrospectively investigated, regarding their subjective response to this pandemic and its clinical relevance.

Results. Of the patients $60.5 \%(n=378)$ experienced greater psychological stress,
$14.5 \%(n=99)$ reported fear of the SARSCoV-2 and the possible danger of infection, $25.5 \%(n=174)$ reported fear resulting from the protective measures taken (lock down) and $4.3 \%(n=29)$ reported fear of both. This differed significantly across diagnoses: people with anxiety disorders reported significantly greater stress as well as greater fear of the virus, whereas people with psychoses were significantly less affected than the other patients. Of the participants $43.7 \%(n=132)$ were so strongly affected that acute treatment had to be implemented and 6.0\% $(n=18)$ had to be referred to inpatient care.
Discussion. People with mental illnesses are particularly vulnerable to the psychological strain of the COVID-19 pandemic. Longterm studies on the further course of disease will be necessary. Additional studies that test interventions to build resilience in this population will also be needed.

Keywords

COVID-19 pandemic - Mental disorder . Psychological stress - Lock down · Crisis intervention
Zeitpunkt der Beschwerde) wurden mithilfe einer Probit-Regression erfasst und jeweils mit der GLM (binomial, probit Familie, in R). Die kardinalskalierten abhängigen Variablen „Anzahl der Kontakte" wurden mithilfe eines T-Tests erfasst und, da im Levene-Test eine Varianzinhomogenität bestand, mit einer robusten Welch-ANOVA ${ }^{[\mathrm{W}]}$ gerechnet.

Ein positives Votum durch die Ethikkommission der Charité - Universitätsmedizin Berlin (EA1/110/20) lag vor.

\section{Ergebnisse}

Die Stichprobe besteht aus 682 im April 2020 in der Psychiatrischen Institutsambulanz der PUK der Charité im SHK Behandelten, darunter 53,1\% $(n=362)$ Frauen und 46,9\% $(n=320)$ Männer (- Tab. 1). Das mittlere Alter betrug 42,70 Jahre (18-87 Jahre; SD=12,94). $39 \%(n=266)$ der Patient*innen hatten zwei psychische Erkrankungen, wurden also in zwei Diagnosegruppen berücksichtigt. 44,3\% $(n=302)$ hatten spontan Belastungen durch die Pandemie an- gegeben: $14,5 \% \quad(n=99)$ litten unter der Angst vor dem Virus SARS-CoV-2, $25,5 \%(n=174)$ unter den Folgen der gesetzlich getroffenen Maßnahmen zur Eindämmung der Pandemie (Kontaktund Besuchsbeschränkungen, Verlust von Tagesstruktur, finanzielle Einbußen oder Unsicherheit etc.), und 4,3\% $(n=29)$ gaben an, unter beidem zu leiden. $6,3 \%(n=43)$ waren durch pandemieunabhängige Faktoren belastet, $28,2 \%$ ( $n=192)$ fühlten sich nicht belastet, und $10,4 \%(n=71)$ fühlten sich in dieser Situation wohler als zuvor (- Tab. 2). 


\section{Originalien}

\begin{tabular}{|c|c|c|c|c|c|c|c|c|c|}
\hline & \multirow[t]{2}{*}{ Gesamt } & \multicolumn{8}{|c|}{ Subjektive Belastung } \\
\hline & & $\begin{array}{l}\text { Fehlende } \\
\text { Angabe }\end{array}$ & $\begin{array}{l}\text { Nicht } \\
\text { belastet }\end{array}$ & $\begin{array}{l}\text { Es geht } \\
\text { mir bes- } \\
\text { ser }\end{array}$ & $\begin{array}{l}\text { Durch } \\
\text { Angst vor } \\
\text { Virus }\end{array}$ & $\begin{array}{l}\text { Durch } \\
\text { Maßnah- } \\
\text { men }\end{array}$ & $\begin{array}{l}\text { Durch } \\
\text { beides }\end{array}$ & $\begin{array}{l}\text { Durch } \\
\text { etwas } \\
\text { anderes }\end{array}$ & \\
\hline Gesamt & $\begin{array}{l}100 \% \\
(n=682)\end{array}$ & $\begin{array}{l}10,8 \% \\
(n=74)\end{array}$ & $\begin{array}{l}28,2 \% \\
n=(192)\end{array}$ & $\begin{array}{l}10,4 \% \\
n=(71)\end{array}$ & $\begin{array}{l}14,5 \% \\
n=(99)\end{array}$ & $\begin{array}{l}25,5 \% \\
n=(174)\end{array}$ & $\begin{array}{l}4,3 \% \\
n=(29)\end{array}$ & $\begin{array}{l}6,3 \% \\
n=(43)\end{array}$ & - \\
\hline Männer & $\begin{array}{l}46,9 \% \\
(n=320)\end{array}$ & $\begin{array}{l}11,9 \% \\
(n=38)\end{array}$ & $\begin{array}{l}28,4 \% \\
(n=91)\end{array}$ & $\begin{array}{l}10,6 \% \\
(n=34)\end{array}$ & $\begin{array}{l}12,2 \% \\
(n=39)\end{array}$ & $\begin{array}{l}27,5 \% \\
(n=88)\end{array}$ & $\begin{array}{l}3,4 \% \\
(n=11)\end{array}$ & $\begin{array}{l}5,9 \% \\
(n=19)\end{array}$ & $\begin{array}{l}x^{2}(6)=4,88 \\
p<0,559\end{array}$ \\
\hline Frauen & $\begin{array}{l}53,1 \% \\
(n=362)\end{array}$ & $\begin{array}{l}9,9 \% \\
(n=36)\end{array}$ & $\begin{array}{l}27,9 \% \\
(n=101)\end{array}$ & $\begin{array}{l}10,2 \% \\
(n=37)\end{array}$ & $\begin{array}{l}16,6 \% \\
(n=60)\end{array}$ & $\begin{array}{l}23,8 \% \\
(n=86)\end{array}$ & $\begin{array}{l}5,0 \% \\
(n=18)\end{array}$ & $\begin{array}{l}6,6 \% \\
(n=24)\end{array}$ & Cramer V $=0,08$ \\
\hline Alter (18-87) & $\begin{array}{l}\text { MW } 42,7 \\
\text { SD } 12,87\end{array}$ & $\begin{array}{l}42,45 \\
S D=11,01\end{array}$ & $\begin{array}{l}43,40 \\
\text { SD } 13,40\end{array}$ & $\begin{array}{l}40,08 \\
\text { SD } 12,21\end{array}$ & $\begin{array}{l}44,72 \\
\text { SD } 12,54\end{array}$ & $\begin{array}{l}41,85 \\
\text { SD } 13,07\end{array}$ & $\begin{array}{l}46,97 \\
\text { SD } 17,01\end{array}$ & $\begin{array}{l}41,67 \\
\text { SD } 12,87\end{array}$ & $\begin{array}{l}\mathrm{F}(5,675)=1,66 \\
p=0,129\end{array}$ \\
\hline $\begin{array}{l}\text { Therapeutische Maß- } \\
\text { nahme ergriffen }\end{array}$ & $\begin{array}{l}29,01 \% \\
(n=181)\end{array}$ & $\begin{array}{l}3,9 \% \\
(n=7)\end{array}$ & $\begin{array}{l}2,2, \% \\
(n=4)\end{array}$ & $\begin{array}{l}5,5 \% \\
(n=10)\end{array}$ & $\begin{array}{l}23,2 \% \\
(n=42)\end{array}$ & $\begin{array}{l}46,4 \% \\
(n=84)\end{array}$ & $\begin{array}{l}3,3 \% \\
(n=6)\end{array}$ & $\begin{array}{l}15,5, \% \\
(n=28)\end{array}$ & $\begin{array}{l}X^{2}(6)=163,94 \\
p<0,0001 \\
\text { Cramer V }=0,49\end{array}$ \\
\hline $\begin{array}{l}\text { Zahl therapeutischer } \\
\text { Kontakte im April } 2020\end{array}$ & $\begin{array}{l}2,03 \\
S D=1,58 \\
(1-20)\end{array}$ & $\begin{array}{l}1,46 \\
S D=0,80 \\
(1-5)\end{array}$ & $\begin{array}{l}1,67 \\
S D=0,95 \\
(1-6)\end{array}$ & $\begin{array}{l}1,97 \\
S D=1,45 \\
(1-6)\end{array}$ & $\begin{array}{l}2,02 \\
\text { SD } 1,27 \\
(1-6)\end{array}$ & $\begin{array}{l}2,45 \\
S D=1,88 \\
(1-16)\end{array}$ & $\begin{array}{l}1,97 \\
S D=1,28 \\
(1-8)\end{array}$ & $\begin{array}{l}3,02 \\
\mathrm{SD}=3,04 \\
(1-20)\end{array}$ & $\begin{array}{l}F^{W}(5,152)=6,501 \\
P^{W}<0,0001\end{array}$ \\
\hline $\begin{array}{l}\text { Klinische Verschlechte- } \\
\text { rung }\end{array}$ & $\begin{array}{l}60,5 \% \\
(n=378)\end{array}$ & $\begin{array}{l}14,7 \% \\
(n=11)\end{array}$ & $\begin{array}{l}0,5 \% \\
(n=1)\end{array}$ & $\begin{array}{l}47,9 \% \\
(n=34)\end{array}$ & $\begin{array}{l}97,0 \% \\
(n=96)\end{array}$ & $\begin{array}{l}96,0 \% \\
(n=167)\end{array}$ & $\begin{array}{l}96,6 \% \\
(n=28)\end{array}$ & $\begin{array}{l}97,7 \% \\
(n=42)\end{array}$ & $\begin{array}{l}x^{2}(12)=971,23 \\
p<0,0001\end{array}$ \\
\hline
\end{tabular}

Bei $60,5 \%(n=378)$ war von den Behandelnden eine klinische Verschlechterung der Symptomatik beschrieben worden, bei $20,8 \%(n=142)$ eine schwere, die pathologische Verhaltensänderungen beinhaltete (- Tab. 3). 29,1\% $(n=181)$ aller Patient*innen hielten wir für so schwer belastet, dass wir gegensteuernde Maßnahmen ergriffen: Von diesen waren $72,9 \%(n=132)$ von der Pandemie belastet, $15,5 \%(n=28)$ durch anderweitige Faktoren und $7,7 \%(n=14)$ derer, die sich subjektiv nicht belastet fanden. Die klinisch leicht oder schwer Belasteten erhielten im April signifikant mehr therapeutische Kontakte als die nicht Belasteten (adjustierter kritischer $p$-Wert 0,03 nach Benjamini und Hochberg; $\mathrm{MW}=2,03$ Kontakte; $\mathrm{SD}=1,58$, WelchTest $\left.\mathrm{F}[5,152.196]=6,501, p^{\mathrm{W}}<0,0001\right)$, der Games-Howell-Post-hoc-Test zeigte hier einen signifikanten Unterschied für die unter den Maßnahmen Leidenden im Vergleich $\mathrm{zu}$ den nicht Leidenden $(0,782, p<0,0001,95 \%$-CI $[0,33,1,24]$; - Tab. 1). 18 Behandelte mussten aufgrund der Schwere der Symptomatik in stationäre Behandlung weitergeleitet werden: Von diesen waren 8 durch die Schutzmaßnahmen und ihre Folgen und
6 durch pandemieunabhängige Faktoren betroffen.

Für Schwere und Art der Belastung bestanden signifikante Zusammenhänge mit den vorbestehenden Diagnosen $\left(\chi^{2}[154]=252,938, p^{* F}=0,003\right.$, Cramer's V=0,23) (•Tab. 2). Für die folgenden Post-hoc-Vergleiche ist der kritische $p$-Werte nach Benjamini und Hochberg adjustiert $(p=0,0055)$. Insgesamt wurde deutlich, dass Angsterkrankte signifikant häufiger Angst vor einer Virusinfektion hatten als vor anderen Belastungen $\left(\chi^{2}[6]=21,410\right.$, $p^{\star \mathrm{F}}=0,002$, Cramer $\left.\mathrm{V}=0,177\right)$. Bei ihnen wurden auch signifikant mehr klinische Verschlechterungen beobachtet $\left(\chi^{2}[1]=12,02, p<0,001\right)$. In den Aufzeichnungen wurde deutlich, dass diese sich häufig zu einer für eine Ansteckung besonders gefährdeten Risikogruppe rechneten, ohne aus medizinischer Sicht $\mathrm{zu}$ einer $\mathrm{zu}$ gehören. Sie behielten die Isolationsmaßnahmen häufig weit über das gesetzlich angeordnete Maß hinaus bei.

Psychotisch Erkrankte gaben signifikant weniger Belastung an und häufiger das Gefühl, es gehe besser als zuvor $\left(\chi^{2}[6]=20,498, p^{* \mathrm{~F}}=0,001\right.$, Cramers $V=0,137)$ und es bestand auch signifikant seltener eine beobachtete Verschlechterung der Psychopathologie $\left(\chi^{2}[1]=18,339, \quad p<0,0001\right)$. Personen mit Persönlichkeitsstörungen litten signifikant häufiger unter Lockdown- und Hygienemaßnahmen und signifikant seltener an Angst vor Infektionen als anders Erkrankte $\left(\chi^{2}[6]=13,070, \quad p^{\star \mathrm{F}}=0,018\right.$, Cramers $\mathrm{V}=0,138)$.

Für diejenigen mit anderen Diagnosen, wie z. B. depressiv, sucht- oder posttraumatisch Erkrankte, konnten wir im Vergleich zu den anderen Diagnosegruppen keine signifikanten Unterschiede in den Belastungen feststellen.

Als besonders prägnante Beschwerden wurden völlige Selbstisolation, körperliche Symptomatik, Impulskäufe, Retraumatisierung, Trinkrezidive, Familienstreitigkeiten und einmal ein Verschwörungswahn beobachtet. 10,8\% $(n=74)$ der Behandelten hatten sich nicht spontan $\mathrm{zu}$ der subjektiven Art ihrer Belastung geäußert - oder dies war nicht dokumentiert worden, und in $8,20 \%(n=56)$ der Fälle war aufgrund der Dokumentation nicht zu entscheiden, ob sich die klinische Symptomatik im Vergleich zu den Vorkontakten verändert hatte. 


\begin{tabular}{|c|c|c|c|c|c|c|c|c|c|}
\hline \multirow{2}{*}{$\begin{array}{l}\text { Diagnosen nach ICD-10 } \\
n=682 \text { Patient*innen } \\
n=179(39 \%) \text { davon } \\
\text { hatten } 2 \text { Diagnosen }\end{array}$} & \multirow{2}{*}{$\begin{array}{l}\text { Gesamt } \\
\text { von } 682\end{array}$} & \multicolumn{7}{|c|}{ Subjektive Belastung } & \multirow[b]{2}{*}{ Statistische Werte } \\
\hline & & $\begin{array}{l}\text { Fehlende } \\
\text { Angaben }\end{array}$ & $\begin{array}{l}\text { Nicht } \\
\text { belastet }\end{array}$ & $\begin{array}{l}\text { Es geht } \\
\text { mir besser }\end{array}$ & $\begin{array}{l}\text { Durch Angst } \\
\text { vor Virus }\end{array}$ & $\begin{array}{l}\text { Durch } \\
\text { Maß- } \\
\text { nahmen }\end{array}$ & $\begin{array}{l}\text { Durch } \\
\text { beides }\end{array}$ & $\begin{array}{l}\text { Durch } \\
\text { etwas } \\
\text { anderes }\end{array}$ & \\
\hline F1: Abhängigkeit & $\begin{array}{l}12,5 \% \\
(n=58)\end{array}$ & $\begin{array}{l}15,3 \% \\
(n=13)\end{array}$ & $\begin{array}{l}29,4 \% \\
(n=25)\end{array}$ & $\begin{array}{l}14,1 \% \\
(n=12)\end{array}$ & $\begin{array}{l}4,7 \% \\
(n=4)\end{array}$ & $\begin{array}{l}29,4 \% \\
(n=25)\end{array}$ & $\begin{array}{l}2,4 \% \\
(n=2)\end{array}$ & $\begin{array}{l}4,7 \% \\
(n=4)\end{array}$ & $\begin{array}{l}X^{2}(6)=11,140 \\
p^{* \mathrm{~F}}=0,053 \\
\text { Cramer } V=0,128\end{array}$ \\
\hline $\begin{array}{l}\text { Darunter: Alkoholabhän- } \\
\text { gigkeit, F10 }\end{array}$ & $\begin{array}{l}5,3 \% \\
(n=36)\end{array}$ & $\begin{array}{l}13,9 \% \\
(n=5)\end{array}$ & $\begin{array}{l}33,3 \% \\
(n=12)\end{array}$ & $\begin{array}{l}8,3 \% \\
(n=3)\end{array}$ & $\begin{array}{l}5,6 \% \\
(n=2)\end{array}$ & $\begin{array}{l}36,1 \% \\
(n=13)\end{array}$ & $\begin{array}{l}2,8 \% \\
(n=1)\end{array}$ & $\begin{array}{l}0 \% \\
(n=0)\end{array}$ & $\begin{array}{l}X^{2}(6)=7,119 \\
p=0,310 \\
\text { Cramer } V=0,102\end{array}$ \\
\hline $\begin{array}{l}\text { F2: psychotisches Syn- } \\
\text { drom }\end{array}$ & $\begin{array}{l}23,5 \% \\
(n=160)\end{array}$ & $\begin{array}{l}15,0 \% \\
(n=24)\end{array}$ & $\begin{array}{l}34,4 \% \\
(n=55)\end{array}$ & $\begin{array}{l}13,1 \% \\
(n=21)\end{array}$ & $\begin{array}{l}11,3 \% \\
(n=18)\end{array}$ & $\begin{array}{l}18,4 \% \\
(n=29)\end{array}$ & $\begin{array}{l}0,6 \% \\
(n=1)\end{array}$ & $\begin{array}{l}7,5 \% \\
(n=12)\end{array}$ & $\begin{array}{l}x^{2}(6)=20,498 \\
p^{* F}=0,001 \\
\text { Cramer } V=0,173\end{array}$ \\
\hline F3: depressives Syndrom & $\begin{array}{l}45,0 \% \\
(n=307)\end{array}$ & $\begin{array}{l}10,4 \% \\
(n=32)\end{array}$ & $\begin{array}{l}28,0 \% \\
(n=86)\end{array}$ & $\begin{array}{l}9,4 \% \\
(n=29)\end{array}$ & $\begin{array}{l}13,7 \% \\
(n=42)\end{array}$ & $\begin{array}{l}27,7 \% \\
(n=85)\end{array}$ & $\begin{array}{l}4,6 \% \\
(n=14)\end{array}$ & $\begin{array}{l}6,2 \% \\
(n=19)\end{array}$ & $\begin{array}{l}x^{2}(6)=2096 \\
p^{* F}=0,916 \\
\text { Cramer } V=0,055\end{array}$ \\
\hline $\begin{array}{l}\text { F4 - 40/41: ängstliches } \\
\text { Syndrom }\end{array}$ & $\begin{array}{l}26,1 \% \\
(n=178)\end{array}$ & $\begin{array}{l}9 \% \\
(n=16)\end{array}$ & $\begin{array}{l}20,8 \% \\
(n=37)\end{array}$ & $\begin{array}{l}7,3 \% \\
(n=13)\end{array}$ & $\begin{array}{l}22,5 \% \\
(n=40)\end{array}$ & $\begin{array}{l}28,7 \% \\
(n=51)\end{array}$ & $\begin{array}{l}7,3 \% \\
(n=13)\end{array}$ & $\begin{array}{l}5,6 \% \\
(n=10)\end{array}$ & $\begin{array}{l}x^{2}(6)=21410 \\
p^{* F}=0,002 \\
\text { Cramer } V=0,177\end{array}$ \\
\hline $\begin{array}{l}\text { F4 - F42/44/45: sonstige } \\
\text { neurotische Syndrome }\end{array}$ & $\begin{array}{l}6,5 \% \\
(n=44)\end{array}$ & $\begin{array}{l}9,1 \% \\
(n=4)\end{array}$ & $\begin{array}{l}20,5 \% \\
(n=9)\end{array}$ & $\begin{array}{l}13,6 \% \\
(n=6)\end{array}$ & $\begin{array}{l}15,9 \% \\
(n=7)\end{array}$ & $\begin{array}{l}22,7 \% \\
(n=10)\end{array}$ & $\begin{array}{l}9,1 \% \\
(n=4)\end{array}$ & $\begin{array}{l}9,1 \% \\
(n=4)\end{array}$ & $\begin{array}{l}X^{2}(6)=5068 \\
p^{* F}=0,463 \\
\text { Cramer } V=0,086\end{array}$ \\
\hline PTSD, F43.1 & $\begin{array}{l}9,7 \% \\
(n=66)\end{array}$ & $\begin{array}{l}6,1 \% \\
(n=4)\end{array}$ & $\begin{array}{l}25,8 \% \\
(n=17)\end{array}$ & $\begin{array}{l}10,6 \% \\
(n=7)\end{array}$ & $\begin{array}{l}12,1 \% \\
(n=8)\end{array}$ & $\begin{array}{l}33,3 \% \\
(n=22)\end{array}$ & $\begin{array}{l}7,6 \% \\
(n=5)\end{array}$ & $\begin{array}{l}4,5 \% \\
(n=3)\end{array}$ & $\begin{array}{l}X^{2}(6)=6,018 \\
p=0,421 \\
\text { Cramer } V=0,138\end{array}$ \\
\hline $\begin{array}{l}\text { F6: Persönlichkeitsstörun- } \\
\text { gen }\end{array}$ & $\begin{array}{l}12,0 \% \\
(n=82)\end{array}$ & $\begin{array}{l}8,5 \% \\
(n=7)\end{array}$ & $\begin{array}{l}28,0 \% \\
(n=23)\end{array}$ & $\begin{array}{l}15,9 \% \\
(n=13)\end{array}$ & $\begin{array}{l}3,7 \% \\
(n=3)\end{array}$ & $\begin{array}{l}31,7 \% \\
(n=25)\end{array}$ & $\begin{array}{l}3,7 \% \\
(n=3)\end{array}$ & $\begin{array}{l}8,5 \% \\
(n=7)\end{array}$ & $\begin{array}{l}x^{2}(6)=13,070 \\
p^{* F}=0,018 \\
\text { Cramer } V=0,138\end{array}$ \\
\hline $\begin{array}{l}\text { Sonstige } \\
\text { F0, F7, F8, F9 }\end{array}$ & $\begin{array}{l}5,9 \% \\
(n=40)\end{array}$ & $\begin{array}{l}10,0 \% \\
(n=4)\end{array}$ & $\begin{array}{l}27,5 \% \\
(n=11)\end{array}$ & $\begin{array}{l}10,0 \% \\
(n=4)\end{array}$ & $\begin{array}{l}12,5 \% \\
(n=5)\end{array}$ & $\begin{array}{l}27,5 \% \\
(n=11)\end{array}$ & $\begin{array}{l}5,0 \% \\
(n=2)\end{array}$ & $\begin{array}{l}7,5 \% \\
(n=3)\end{array}$ & $\begin{array}{l}X^{2}(6)=0,417 \\
P^{* F}=0,996 \\
\text { Cramer } V=0,025\end{array}$ \\
\hline
\end{tabular}

Für Geschlecht bestanden weder bezüglich Stärke $\left(\chi^{2}[2]=0,95, p^{\star \mathrm{F}}=0,622\right)$ noch bezüglich Art der Belastung $\left(\chi^{2}[6]\right.$ $\left.=4,88, p^{* \mathrm{~F}}=0,559\right)$ signifikante Unterschiede. Das gleiche gilt für die Variable „Alter" bezüglich Stärke $(Z=0,066$, $p=0,947)$ und Art der Belastung $(\mathrm{Z}=$ $-0,553, p=0,580)$, und auch für die Variable "Zeitpunkt der Äußerung“ - vor oder nach begonnener Lockerung der Maßnahmen - bezüglich der Stärke $(Z=-0,200, p=0,841)$ und der Art der Belastung $(Z=0,649, p=0,516$; - Tab. 1). Über die Art der klinischen Dekompensation konnten wir bei nur $4,96 \%(n=34)$ eine konkrete Beschreibung sichern, darunter 5 Trinkrezidive, 5 eskalierende Familienkonflikte und 2 Retraumatisierungen.

Neun Patient ${ }^{*}$ innen wurden aufgrund der aktuellen psychischen Belastung erst- mals in psychiatrisch-psychotherapeutische Behandlung aufgenommen; von diesen litten 6 unter eine depressiven, 2 unter einer ängstlichen Symptomatik und eine Person an einer Alkoholerkrankung.

\section{Diskussion}

Die Ergebnisse unserer Studie bestätigen diagnoseabhängige signifikante Unterschiede in der Zunahme krankheitswertiger Symptomatik bei psychisch erkrankten Menschen in den ersten Wochen der COVID-19-Pandemie und der von ihnen subjektiv wahrgenommenen Ursache der Belastung. Bei 60,5\% unserer Stichprobe beobachteten wir eine klinische Verschlechterung im Vergleich zum unmittelbaren Vorkontakt; 44,3\% $(n=302)$ gaben von sich aus spontan an, dass sie diese Verschlechterung subjektiv als durch die Pandemie bedingt erlebten. Anders als in einer großen Stichprobe chinesischer Allgemeinbevölkerung beobachtet, konnten wir in unserer Stichprobe für Frauen keine signifikant häufigere Angst vor dem Virus beschreiben [39]. Jedoch konnten wir die Beobachtung, dass die subjektive Wahrnehmung eigener schlechter Gesundheit den psychischen Stress erhöht [5], bei vielen Menschen mit einer Angsterkrankung bestätigen.

Während in Italien für die psychisch gesunde ältere Allgemeinbevölkerung eine größere Resilienz gegenüber einer psychischen Belastung durch die Pandemie beschrieben wurde [7] und in Frankreich eher eine höhere Vulnerabilität für ältere Personen mit psychischer Vorerkrankung identifiziert wurde [6], sahen wir 
Tab. 3 Ausprägung der klinischen Verschlechterung unter Berücksichtigung der psychischen Grunderkrankung

\begin{tabular}{|c|c|c|c|c|c|}
\hline \multirow{2}{*}{$\begin{array}{l}\text { Diagnosen nach ICD10 } \\
n=682 \text { Patient*innen } \\
n=179(39 \%) \text { davon hatten } 2 \text { Diagnosen }\end{array}$} & \multicolumn{5}{|c|}{ Klinische Verschlechterung } \\
\hline & Gesamt & Nicht & Leicht & Schwer & Statistische Werte \\
\hline F1: Abhängigkeit & $\begin{array}{l}100 \% \\
(n=78)\end{array}$ & $\begin{array}{l}44,9 \% \\
(n=35)\end{array}$ & $\begin{array}{l}34,9 \% \\
(n=27)\end{array}$ & $\begin{array}{l}20,5 \% \\
(n=16)\end{array}$ & $\begin{array}{l}x^{2}(2)=1111 \\
p^{* F}=0,372\end{array}$ \\
\hline Darunter: Alkoholabhängigkeit, F10 & $\begin{array}{l}100 \% \\
(n=33)\end{array}$ & $\begin{array}{l}42,2 \% \\
(n=14)\end{array}$ & $\begin{array}{l}27,3 \% \\
(n=9)\end{array}$ & $\begin{array}{l}30,3 \% \\
(n=10)\end{array}$ & $\begin{array}{l}X^{2}(2)=1981 \\
p=0,576\end{array}$ \\
\hline F2: psychotisches Syndrom & $\begin{array}{l}100 \% \\
(n=140)\end{array}$ & $\begin{array}{l}56,0 \% \\
(n=77)\end{array}$ & $\begin{array}{l}27,1 \% \\
(n=38)\end{array}$ & $\begin{array}{l}17,9 \% \\
(n=25)\end{array}$ & $\begin{array}{l}x^{2}(2)=18.454 \\
p^{* F}<0,001\end{array}$ \\
\hline F3: depressives Syndrom & $\begin{array}{l}100 \% \\
(n=263)\end{array}$ & $\begin{array}{l}36,0 \% \\
(n=102)\end{array}$ & $\begin{array}{l}39,9 \% \\
(n=113)\end{array}$ & $\begin{array}{l}24,0 \% \\
(n=48)\end{array}$ & $\begin{array}{l}X^{2}(2)=2478 \\
p^{* F}=0,290\end{array}$ \\
\hline F4 - 40/41: ängstliches Syndrom & $\begin{array}{l}100 \% \\
(n=164)\end{array}$ & $\begin{array}{l}28,0 \% \\
(n=46)\end{array}$ & $\begin{array}{l}43,3 \% \\
(n=71)\end{array}$ & $\begin{array}{l}28,7 \% \\
(n=47)\end{array}$ & $\begin{array}{l}x^{2}(2)=12465 \\
p^{* F}=0,002\end{array}$ \\
\hline F4 - 44/45: sonstige neurotische Syndrome & $\begin{array}{l}100 \% \\
(n=31)\end{array}$ & $\begin{array}{l}36,6 \% \\
(n=15)\end{array}$ & $\begin{array}{l}34,1 \% \\
(n=14)\end{array}$ & $\begin{array}{l}29,3 \% \\
(n=12)\end{array}$ & $\begin{array}{l}X^{2}(2)=1064 \\
p^{* F}=0,587\end{array}$ \\
\hline PTSD, F43.1 & $\begin{array}{l}100 \% \\
(n=61)\end{array}$ & $\begin{array}{l}32,8 \% \\
(n=20)\end{array}$ & $\begin{array}{l}41,0 \% \\
(n=25)\end{array}$ & $\begin{array}{l}26,2 \% \\
(n=16)\end{array}$ & $\begin{array}{l}X^{2}(2)=1,290 \\
p=0,524\end{array}$ \\
\hline F6: Persönlichkeitsstörungen & $\begin{array}{l}100 \% \\
(n=78)\end{array}$ & $\begin{array}{l}46,2 \% \\
(n=36)\end{array}$ & $\begin{array}{l}35,9 \% \\
(n=28)\end{array}$ & $\begin{array}{l}17,9 \% \\
(n=14)\end{array}$ & $\begin{array}{l}X^{2}(2)=2017 \\
p^{* F}=0,365\end{array}$ \\
\hline Sonstige F0, F7, F8, F9 & $\begin{array}{l}100 \% \\
(n=35)\end{array}$ & $\begin{array}{l}36,1 \% \\
(n=13)\end{array}$ & $\begin{array}{l}44,4 \% \\
(n=16)\end{array}$ & $\begin{array}{l}19,4 \% \\
(n=7)\end{array}$ & $\begin{array}{l}X^{2}(2)=0,734 \\
P^{* F}=0,593\end{array}$ \\
\hline Frauen & $\begin{array}{l}100 \% \\
(n=334)\end{array}$ & $\begin{array}{l}38,3 \% \\
(n=128)\end{array}$ & $\begin{array}{l}40,1 \% \\
(n=134)\end{array}$ & $\begin{array}{l}21,6 \% \\
(n=72)\end{array}$ & $\begin{array}{l}X^{2}(2)=1,68 \\
P^{* F}=0,432\end{array}$ \\
\hline Männer & $\begin{array}{l}100 \% \\
(n=290)\end{array}$ & $\begin{array}{l}40,7 \% \\
(n=118)\end{array}$ & $\begin{array}{l}35,2 \% \\
(n=102)\end{array}$ & $\begin{array}{l}24,1 \% \\
(n=79)\end{array}$ & \\
\hline Gesamt & $\begin{array}{l}100 \% \\
(n=624)\end{array}$ & $\begin{array}{l}39,4 \% \\
(n=246)\end{array}$ & $\begin{array}{l}37,8 \% \\
(n=236)\end{array}$ & $\begin{array}{l}22,7 \% \\
(n=142)\end{array}$ & $\begin{array}{l}\text { Keine Angaben: } \\
n=58\end{array}$ \\
\hline
\end{tabular}

in unserer Stichprobe keinen signifikanten Zusammenhang zwischen Lebensalter und Art oder Stärke der Belastung durch die Pandemie. Offenbar bestehen in Deutschland im Vergleich zu anderen Ländern sowohl bezüglich der sozialen Sicherheit schwer psychisch Erkrankter als auch körperlicher Gesundheit keine so gravierenden altersabhängigen Unterschiede in der subjektiv erlebten Gefährdung durch Vorerkrankungen.

Auch konnten wir keine geschlechtsabhängigen Unterschiede in Zusammenhang mit Art oder Stärke der Belastung bestätigen [7, 8]. Möglicherweise hängt das damit zusammen, dass in unserer Stichprobe - mit einem hohen Anteil chronisch psychisch erkrankter Menschen - rollenspezifische Unterschiede eine geringere Rolle spielen als in der Allgemeinbevölkerung (z.B. bezüglich Versorgung von Kindern, Sorge um Arbeitsplatz etc.).
Eine signifikante Verschlechterung bei der Gruppe derjenigen, die unter einer PTSD litten, konnten wir nicht beobachten: Lediglich 2 Personen mit vorbestehender PTSD erlitten durch Pandemiemaßnahmen eine Retraumatisierung. Von den 9 uns neu zugewiesenen Patient ${ }^{*}$ innen litt keine ${ }^{\star} r$ unter einer PTSD. Die im Vergleich zu den Studien aus Italien, China, USA und Tunesien [7, 11, 20, 40] geringe Bedeutung einer Traumatisierung in unserer Studienpopulation psychisch erkrankter Menschen in Deutschland könnte auch im Zusammenhang damit stehen, dass in weiten Teilen Deutschlands bislang ein blander Pandemieverlauf erlebt wurde.

Interessant bleibt die Frage, was die psychische Widerstandsfähigkeit der Menschen - hier also insbesondere psychisch erkrankte Menschen - befähigt, schwierige Lebenssituationen ohne anhaltende Beeinträchtigung zu über- stehen. So empfehlen mehrere Autoren, die Resilienzfähigkeit dieser Subpopulation zu testen [24] und zu fördern [38]. Für das gute Überstehen einer schweren psychischen Belastung wurden für die gesunde Allgemeinbevölkerung verschiedene angeborene und erworbene Faktoren, u. a. aktiv bleiben, Belastungen als Wachstumschance begreifen können, Optimismus, Sinn für Humor, Arbeit und Liebe genannt [19]. Ein gutes Selbstbild helfe, übrigens ebenso wie ein gemeinsamer Widerstand gegen einen äußeren Feind, eine Quarantäne zu überstehen [5]: Alles Eigenschaften, die psychisch Erkrankten zumindest in einer Krankheitsphase nicht oder vermindert zur Verfügung stehen.

Aus Italien wurde berichtet, dass diejenigen, die sich in Quarantäne befunden hatten, signifikant weniger Stresssymptomatik hatten als die restliche Allgemeinbevölkerung - allerdings hatten 
diese während der Quarantäne auch psychologische Unterstützung erhalten [23]. Stress sei mit sozialer Unterstützung sehr viel besser zu verarbeiten als ohne, dyadisches Coping in der (intakten) Partnerbeziehung am hilfreichsten. Unter den auf Intensivstationen in den USA Beschäftigte wurden feste Paare gegenseitiger Unterstützung geschaffen, ein „peer support“ ähnlich einem in der US-Armee üblichen, den „battle buddies“ [1]. Die Krisentelefone [36] oder anonymen telefonischen Gesprächsangebote für Senior*innen [29] sind vorhandene Hilfsangebote, die psychologische Unterstützung anbieten und Vereinsamten telefonische Gesprächspartner*innen vermitteln können. Für chronisch psychiatrisch Erkrankte, die oft sehr viel eingeschränkter sind in der Fähigkeit, Hilfe aufzusuchen, Vertrauen zu bilden, Kontakt aufzunehmen, und die im Alltag sehr oft allein leben, sind diese Angebote nur selten realisierbar.

Hier wurde die Bedeutung der therapeutischen Beziehung deutlich [30], denn wir konnten viele Betroffene durch die Erhöhung von Frequenz und Intensität der therapeutischen Gesprächsangebote stabilisieren. Allerdings forderte dieses Vorgehen sehr viel Energie und Zeit. Deutlich wird, dass der ambulante psychiatrische Sektor in Pandemiezeiten ebenfalls als dringend personell $\mathrm{zu}$ verstärkender Gesundheitsbereich gedacht werden sollte. Zur Förderung der psychischen Stabilität der Betroffenen könnte das Prinzip des partnerschaftlichen „peer support" eine Methode oder Maßnahme sein, die in der psychiatrischen Versorgung chronisch Erkrankter künftig mitgedacht werden sollte.

Neben dem unterstützenden sozialen Kontakt werden noch einige weitere Faktoren genannt, die psychische Widerstandsfähigkeit in Pandemiezeiten förderten: geringes Risiko sich anzustecken, große Hoffnung auf das eigene Überleben, hohes Vertrauen in Ärzte und Wissen über Virus und Erkrankung [34, 40]. In vertrauensvollem Setting vermittelte valide Kenntnisse über Ansteckungswege und sinnvolle Schutzmaßnahmen, Krankheitsverlauf bei Ansteckung [42] sowie Anleitung gesundheitsförderliche Maßnahmen („unbedingt einmal am Tag
0,5 h Spazieren gehen“) helfe, weniger durch das Geschehen belastet zu werden.

Interessant bleibt, dass es in unserer Stichprobe einige Menschen gab, die die soziale Distanzierung subjektiv als angenehm empfanden und es genossen, mehr Ruhe zu haben, weniger Lärm und Unruhe auf den Straßen, keine belastenden Anfragen von Jobcenter und anderen Ämtern - signifikant häufiger waren dies Menschen, die eine Psychose erlebt hatten oder erleben. Bei ihnen war auch von den Behandelnden im Aktenverlauf eine geringere klinische Belastung dokumentiert worden. Anderseits waren auch 8 von den 18 Patient ${ }^{*}$ innen, die im April 2020 so schwer dekompensierten, dass sie stationär aufgenommen werden mussten, psychotisch vorerkrankt. Hier sind Vergleichsbeobachtungen zu einem Äquivalenzzeitraum ohne Pandemie notwendig, um dies abschließend beurteilen zu können. Dasselbe gilt für die von uns in diesem Zeitrahmen beobachteten Trinkrezidive bei 5 von 36 zuvor abstinenten alkoholabhängig Erkrankten.

Unsere Untersuchungsergebnisse sind dadurch limitiert, dass wir in $10,8 \%$ $(n=74)$ der Fälle die Art und in 8,20\% $(n=58)$ der Fälle die Schwere der Belastung nicht ermitteln konnten. Über die Art der klinischen Dekompensation konnten wir aufgrund fehlender Vergleichsdaten keine statistisch valide Aussage machen, hier sind weitere Untersuchungen erforderlich. Eine weitere Limitation unserer Studie ist, dass sie nur einen sehr kurzen Zeitraum berücksichtigt. Da die Untersuchung retrospektiv erfolgte, konnten wir auch nur die deutlich schriftlich dokumentierten Beschwerden der Betroffenen aufnehmen. Auch konnten wir keine standardisierten Fragebogeninstrumente verwenden, weshalb sich unsere Daten nicht mit den bisherigen Studien - z.B. in der Allgemeinbevölkerung - vergleichen lassen. Ebenso konnten in unserer Datenbank einige weitere, potenziell relevante Faktoren - wie z.B. sozioökonomischer Status und kultureller Hintergrund nicht quantifiziert werden, Aspekte, die in künftigen Studien berücksichtigt werden könnten. Auch sind weitere Studien mit größeren Stichproben zur Beurteilung des längeren Verlaufes sowohl bei psychisch Erkrankten als auch der pandemiebedingten Entwicklung psychischer Erkrankungen in der bislang gesunden Bevölkerung erforderlich.

Durch unsere Studie wird deutlich, dass das weitere Erforschen und Entwickeln von Maßnahmen zur Förderung von der Resilienz bei psychisch schwer Erkrankten vordringlich bleibt. Themen wie „peer support" könnten weitergedacht, evaluiert und ggf. implementiert werden. Zudem gibt unsere Studie wertvolle Hinweise darauf, welche Patient ${ }^{*}$ innengruppen besonders bei künftigen Pandemien durch präventive Maßnahmen besser geschützt werden können.

\section{Schlussfolgerungen für die Praxis}

- Psychiatrisch Erkrankte sind bei erneuten seuchengesetzlichen Kontaktbeschränkungen besonders zu berücksichtigen, $z$. B. indem soziale therapeutische Angebote auch unter rigiderem Hygienekonzept zeitnah möglich gemacht werden (Spaziergänge, Gruppen im Freien, sportliche Aktivitäten ect.).

- Bei erneutem Infektionsgeschehen und Lockdown sollte der Kontakt zu psychisch Vorerkrankten aktiv gesucht werden (telefonisch, per Video gestützt, analog, ggf. Hausbesuche).

- Eine personelle Verstärkung ambulanter psychiatrischer Einrichtungen sollte in der medizinischen Planung bei globalen Bedrohungen berücksichtigt werden, denn in der sog. ersten Welle der COVID-19Pandemie wurde mit 60,5\% ein großer Teil der Patient*innen einer psychiatrischen Ambulanz klinisch belastet, bei 29,1\% der Behandelten wurden therapeutische Maßnahmen erforderlich.

- Resilienzforschung und -förderung für psychisch Erkrankte ist dringend erforderlich. 


\section{Korrespondenzadresse

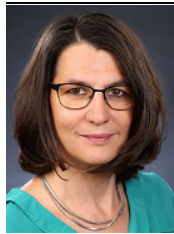 \\ Johanna G. Winkler \\ Psychiatrische Institut- sambulanz der Charité im St. Hedwig-Krankenhaus, Charité - Universitätsmedizin Berlin Große Hamburger Str. 5-11, 10115 Berlin, Deutschland johanna.winkler@charite.de}

Funding. Open Access funding enabled and organized by Projekt DEAL.

\section{Einhaltung ethischer Richtlinien}

Interessenkonflikt. J.G. Winkler, D. Jalilzadeh Masah, J.K. Moran, J. Bretz, I. Tsagkas, T. Goldschmidt und M. Schouler-Ocak geben an, dass kein Interessenkonflikt besteht.

Für diesen Beitrag wurden von den Autoren keine Studien an Menschen oder Tieren durchgeführt. Für die aufgeführten Studien gelten die jeweils dort angegebenen ethischen Richtlinien.

Open Access. Dieser Artikel wird unter der Creative Commons Namensnennung 4.0 International Lizenz veröffentlicht, welche die Nutzung, Vervielfältigung, Bearbeitung, Verbreitung und Wiedergabe in jeglichem Medium und Format erlaubt, sofern Sie den/die ursprünglichen Autor(en) und die Quelle ordnungsgemäß nennen, einen Link zur Creative Commons Lizenz beifügen und angeben, ob Änderungen vorgenommen wurden.

Die in diesem Artikel enthaltenen Bilder und sonstiges Drittmaterial unterliegen ebenfalls der genannten Creative Commons Lizenz, sofern sich aus der Abbildungslegende nichts anderes ergibt. Sofern das betreffende Material nicht unter der genannten Creative Commons Lizenz steht und die betreffende Handlung nicht nach gesetzlichen Vorschriften erlaubt ist, ist für die oben aufgeführten Weiterverwendungen des Materials die Einwilligung des jeweiligen Rechteinhabers einzuholen.

Weitere Details zur Lizenz entnehmen Sie bitte der Lizenzinformation auf http://creativecommons.org/ licenses/by/4.0/deed.de.

\section{Literatur}

1. Albott CS, Wozniak JR, McGlinch BP, Wall MH, Gold BS, Vinogradov S (2020) Battle buddies: rapid deployment of a psychological resilience intervention for health care workers during the COVID-19 pandemic. Anesth Analg 131(1):43-54

2. Beltz.Statistik und Forschungsmethoden - Lehrbuch. Mit Online-Material - Michael Eid, Mario Gollwitzer, Manfred Schmitt | BELTZ. . Im Internet: https://www.beltz.de/fachmedien/psychologie/ buecher/produkt produktdetails/8413-statistik und_forschungsmethoden.html; Stand: 1. Dez. 2020
3. Berthelot N, Lemieux R, Garon-Bissonnette J et al (2020) Uptrend in distress and psychiatric symptomatology in pregnant women during the coronavirus disease 2019 pandemic. Acta Obstet Gynecol Scand 99:848-855

4. Bortz J, Doering N (2006) Forschungsmethoden und Evaluation für Human- und Sozialwissenschaftler, 4. Aufl. Springer, Berlin Heidelberg https://doi.org/10.1007/978-3-540-33306-7 (Limitierte Sonderausgabe)

5. Cazes C, Rosnet E, Bachelard C et al (1996) Group dynamics during the EXEMSI isolation study. Experimental Campaign for the European Manned Space Infrastructure. Adv Space Biol Med 5:245-262

6. Chevance A, Gourion D, Hoertel N et al (2020) Ensuring mental health care during the SARSCoV-2 epidemic in France: a narrative review. Encephale 46:193-201

7. Costantini A, Mazzotti E (2020) Italian validation of CoViD-19 Peritraumatic Distress Index and preliminary data in a sample of general population. Riv Psichiatr 55:145-151

8. Di Crosta A, Palumbo R, Marchetti D, Ceccato I, La Malva P, Maiella R et al Individual differences, economic stability, and fear of contagion as risk factors for PTSD symptoms in the COVID19 emergency. Front Psychol [Internet]. 8. September 2020. https://www.ncbi.nlm.nih.gov/ pmc/articles/PMC7506146/. Zugegriffen: 9. Nov. 2020

9. Die COVID-19-Pandemie veränderte nicht die Zahl, aber die Art psychiatrischer Notfälle. springermedizin.de. https://www.springermedizin.de/ covid-19/psychiatrische-notfaelle/die-covid-19pandemie-veraenderte-nicht-die-zahl-aber-dieart-ps/18213060?fulltextView=true; Zugegriffen: 1.Dez. 2020

10. Fehlende Tagesstruktur für psychisch kranke Menschen in Zeiten der Corona-Krise - Diakonie Schweinfurt. https://www.diakonie-schweinfurt de/fehlende-tagesstruktur-fuer-psychischkranke-menschen-in-zeiten-der-corona-krise. Zugegriffen: 8. Juli 2020

11. Fekih-Romdhane F, Ghrissi F, Abbassi B et al (2020) Prevalence and predictors of PTSD during the COVID-19 pandemic:findings froma Tunisian community sample. Psychiatry Res 290:113131

12. Fovet T, Lancelevée C, Eck M et al (2020) Mental health care in French correctional facilities during the Covid-19 pandemic. Encephale 46:S60-S65

13. Ghoshal R (2020) Twin public health emergencies: Covid-19 and domestic violence. Indian J Med Ethics. https://ijme.in/articles/twin-publichealth-emergencies-covid-19-and-domesticviolence/?galley $=\mathrm{html}$. Zugegriffen: 6. Jan. 2021

14. Halouani N, Turki M, Ennaoui R et al (2018) Psychological distress in medical and paramedical personnel in anesthesia and intensive care. Pan Afr Med J 29:221

15. Hao F, Tan W, Jiang L, Zhang L, Zhao X, Zou Y et al (2020) Do psychiatric patients experience more psychiatric symptoms during COVID-19 pandemic and lockdown? A case-control study with service and research implications for immunopsychiatry. Brain Behav Immun 87:100-106

16. Hassan $G$, Ventevogel $P$, Jefee-Bahloul $H$ et al (2016) Mental health and psychosocial wellbeing of Syrians affected by armed conflict. Epidemiol Psychiatr Sci 25:129-141

17. Heim M (2020) Protokoll aus Berliner Psychiatrie: "Das Virus als Wahninhalt". Die Tageszeitung: taz. https://taz.de/!5686038/.Zugegriffen:8.Juli 2020
18. Jesuthasan J, Sönmez E, Abels I et al (2018) Neardeath experiences, attacks by family members, and absence of health care in their home countries affect the quality of life of refugee women in Germany: a multi-region, cross-sectional, gendersensitive study. BMCMed 16:15

19. Lecic-Tosevski D, Vukovic O, Stepanovic J (2011) Stress and personality. Psychiatriki 22:290-297

20. Liu CH, Zhang E, Wong GTF et al (2020) Factors associated with depression, anxiety, and PTSD symptomatology during the COVID-19 pandemic: clinical implications for U.S. young adult mental health. Psychiatry Res 290:113172

21. Martinkevich P, Larsen LL, Græsholt-Knudsen T et al (2020) Physical child abuse demands increased awareness during health and socioeconomic crises like COVID-19. Acta Orthop. https://doi.org/10. 1080/17453674.2020.1782012

22. Mazza M, Marano G, Lai C et al (2020) Danger in danger: interpersonal violence during COVID-19 quarantine. Psychiatry Res 289:113046

23. Meuwly N, Bodenmann G, Germann J et al (2012) Dyadic coping, insecure attachment, and cortisol stress recovery following experimentally induced stress. J Fam Psychol 26:937-947

24. Muller L, Spitz E (2003) Multidimensional assessment of coping: validation of the BriefCOPEamong French population. Encephale 29:507-518

25. Nochaiwong S, Ruengorn C, Awiphan Ret al (2020) Mental health circumstances among health care workers and general public under the pandemic situation of COVID-19(HOME-COVID-19). Medicine 99:e20751

26. Nussbaumer-Streit B, Mayr V, Dobrescu Al et al (2020) Quarantine alone or in combination with other public health measures to control COVID19: a rapid review. Cochrane Database Syst Rev. https://doi.org/10.1002/14651858.CD013574

27. Roesch E, Amin A, Gupta J et al Violence against women during covid-19 pandemic restrictions. BMJ 2020; 369. https://www.bmj.com/content/ 369/bmj.m1712. Zugegriffen: 8. Juli 2020

28. Röhr S, Müller F, Jung F et al (2020) Psychosocial impact of quarantine measures during serious coronavirus outbreaks: a rapid review. Psychiatr Prax 47:179-189

29. Silbernetz Gemeinsam gegen Einsamkeit im Alter. https://www.silbernetz.org/. Zugegriffen: 8. Juli 2020

30. Soumaki E, Anagnostopoulos DC (2018) Psychoanalytic psychotherapy in times of social crisis: the impact on therapeutic relationship. Psychiatriki 29:257-263

31. Spitzer M (2020) Psychologie und Pandemie. Nervenheilkunde 39:274-283

32. SwartE, IhleP,Gothe H, MatusiewiczD(Hrsg) (2014) Routinedaten im Gesundheitswesen. Handbuch Sekundärdatenanalyse: Grundlagen, Methoden und Perspektiven. Huber, Bern

33. Taioli E, Tuminello S, Lieberman-Cribbin W et al (2018) Mental health challenges and experiences in displaced populations following Hurricane Sandy and Hurricane Harvey: the need for more comprehensive interventions in temporary shelters. J Epidemiol Community Health 72:867-870

34. Tarantelli CB (2003) Life within death: towards a metapsychology of catastrophic psychic trauma. Int JPsychoanal 84:915-928

35. Tariku M, Hajure M (2020) Available evidence and ongoing hypothesis on corona virus (COVID19) and psychosis: is corona virus and psychosis related? A narrative review. Psychol Res Behav 
Manag 13:701-704. https://doi.org/10.2147/ PRBM.S264235

36. Stiftung Deutsche Depressionshilfe Überregionale Krisentelefone. https://www.deutschedepressionshilfe.de/krisentelefone. Zugegriffen 8. Juli 2020

37. Vergara Serrano JC, Garrido SI (2014) Estrés crónico y alteraciones psiquiátricas como factor de riesgo en gripe A. Med Intensiva 38:528-529

38. Vinkers CH, van Amelsvoort T, Bisson Jl et al (2020) Stress resilience during the coronavirus pandemic. Eur Neuropsychopharmacol 35:12-16

39. Wang C, Pan R, Wan X et al (2020) Immediate psychological responses and associated factors during the initial stage of the 2019 Coronavirus disease (COVID-19) epidemic among the general population in China. Int J Environ Res Public Health 17. https://doi.org/10.3390/ijerph17051729

40. Wang C, Pan R, Wan X et al (2020) A longitudinal study on the mental health of general population during the COVID-19 epidemic in China. Brain Behav Immun 87:40-48

41. Zeidler J, Braun S (2012) Sekundärdatenanalysen. In: Schöffski O, Graf von der Schulenburg JM (Hrsg) Gesundheitsökonomische Evaluationen, 4. Aufl. Springer, Berlin Heidelberg https://doi.org/10. 1007/978-3-642-21700-5

42. Zhou S-J, Zhang L-G, Wang L-L et al (2020) Prevalence and socio-demographic correlates of psychological health problems in Chinese adolescents during the outbreak of COVID-19. Eur Child Adolesc Psychiatry 29:749-758

43. Zhu J, Fang F, Sjölander A et al (2017) Firstonset mental disorders after cancer diagnosis and cancer-specific mortality: a nationwide cohort study. Ann Oncol 28:1964-1969

\section{Fachnachrichten}

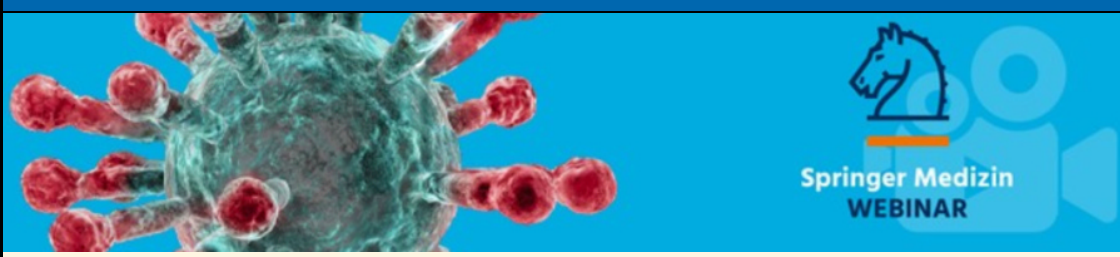

\section{Corona-Webinare bei Springer Medizin}

\section{Webinare und Videointerviews über das Ärzteportal SpringerMedizin.de}

Springer Medizin hat seit April die Initiative Corona-Webinar über sein Ärzteportal (www.springermedizin.de) gestartet. Ärzte, die auf diesem Portal registriert sind, gelangen über Videoseminare an kostenloses Wissen rund um das Corona-Virus. In jedem Webinar oder Videointerview kommt ein Experte zu einem bestimmten Thema zu Wort und gibt fachlich gesichertes Wissen an Kollegen weiter, die sich mit Symptomatik, Diagnostik und Therapie in Klinik und Praxis beschäftigen. Wöchentlich kommen neue Themen hinzu!

Auf der zentralen Corona-Update-Seite auf SpringerMedizin.de sind unter anderem bereits folgende Webinare verfügbar:

- COVID-19: Was wir heute wissen und wie es weitergehen könnte

- "Live" aus dem Sektionssaal Pathologische Befunde bei COVID-19

- COVID-19 überstanden - der mühsame Weg zurück ins Leben

- Zurück zur Routine? Wie meistern wir zukünftige Pandemien?

- Wie kann sich medizinisches Personal vor einer Ansteckung schützen?

- Der Corona-Patient zwischen Hausarztpraxis und Corona-Ambulanz

- Geriatrie und COVID-19: Wo wir stehen und welche Herausforderungen es gibt

- Versorgung psychisch Erkrankter während und nach der Coronapandemie

- Neurologische Manifestationen bei COVID-19: Was bisher bekannt ist
Alle Webinare sind über www.springermedizin.de/webinare zugänglich, die Seite wird ständig aktualisiert und erweitert.

„In unserer Ärztekommunikation werden wir das Webinar-Format zukünftig stärker nutzen, denn es ist eine sehr wertvolle Ergänzung zu den bestehenden Print- und OnlineAngeboten", erläutert Cécile Mack, Director Digital Product \& Marketing von Springer Medizin. „Die Inhalte eines Webinars oder VideoInterviews können durch die direkte Ansprache schneller aufgenommen werden. Unsere Redakteure der verschiedenen Magazine und Zeitschriften laden zum Ausbau dieser Initiative ausgewählte Autoren und Herausgeber ein, ihr Fachwissen zu Corona zu teilen. Neben den aktuellen Aspekten werden wir auch in den nächsten Monaten das Thema eng begleiten und über die Erkenntnisse aus klinischen Studien sowie der Entwicklung von Therapieoptionen und Impfstoffen berichten. Solange die SARS-CoV-2/Covid-19-Pandemie eine Bedrohung unserer Gesellschaft darstellt, wird Springer Medizin als ein führender Marktvertreter diese Inhalte kostenfrei vor der Bezahlschranke platzieren, um allen Ärzten eine sichere und fundierte Wissensbasis zu geben. Denkbar ist außerdem, dieses Format auch auf andere medizinische Themen oder zusätzliche Fachgebiete auszudehnen", so die Digitaldirektorin. 